\title{
Planar Reorientation Maneuvers of Space Multibody Systems Using Internal Controls
}

\author{
Mahmut Reyhanoglu* and N. Harris McClamroch $\dagger$ \\ University of Michigan, Ann Arbor, Michigan 48109
}

\begin{abstract}
In this paper a reorientation maneuvering strategy for an interconnection of planar rigid bodies in space is developed. It is assumed that there are no exogeneous torques, and torques generated by joint motors are used as means of control so that the total angular momentum of the multibody system is a constant, assumed to be zero in this paper. The maneuver strategy uses the nonintegrability of the expression for the angular momentum. We demonstrate that large-angle maneuvers can be designed to achieve an arbitrary reorientation of the multibody system with respect to an inertial frame. The theoretical background for carrying out the required maneuvers is briefly summarized. Specifications and computer simulations of a specific reorientation maneuver, and the corresponding control strategies, are described.
\end{abstract}

\section{Introduction}

I $\mathrm{N}$ this paper we develop a reorientation strategy for a system of $N$ planar rigid bodies in space that are interconnected by ideal frictionless pin joints in the form of an open kinematic chain. Angular momentum preserving controls, e.g., torques generated by joint motors, are considered. The $N$-body system is assumed to have zero initial angular momentum. Our earlier work ${ }^{1,2}$ demonstrated that reorientation of a planar multibody system with three or more interconnected bodies using only joint torque inputs is an inherently nonlinear control problem that is not amenable to classical methods of nonlinear control. The goal of this study is to indicate how control strategies can be explicitly constructed to achieve the desired absolute reorientation of the $N$-body system.

There are many physical advantages in using internal controls, e.g., joint torque controls, to carry out the desired multibody reorientation maneuvers. First of all, this control approach does not modify the total angular momentum of the multibody system. In addition, internal controls have obvious advantages in terms of energy conservation. Moreover, they can be implemented using standard electrical servo motors, a simple and reliable control actuator technology.

The formal development in this paper is concerned with control of a multibody interconnection in space that has zero angular momentum. Although these results are formulated in a general setting, we have been motivated by several classes of specific problems. Several potential applications of our general results are now described.

Manipulators mounted on space vehicles and space robots have been envisioned to carry out construction, maintenance, and repair tasks in an external space environment. These space systems are essentially multibody systems satisfying the assumptions of this paper. To carry out the desired tasks, they must be capable of performing a variety of reorientation maneuvers. Previous research on maneuvering of such space multibody systems has mainly focused on maneuvers that achieve desired orientation of some of the bodies, e.g., an end effector, whereas the orientation of some of the remaining bodies cannot be specified, at least using the methodologies employed ${ }^{3-8}$ Using the approach suggested in this paper, maneuvers that achieve any desired reorientation for all of the

Received June 25, 1991; revision received Feb. 4, 1992; accepted for publication Feb. 28, 1992. Copyright (C) 1992 by the American Institute of Aeronautics and Astronautics, Inc. All rights reserved.

*Professor, Department of Aerospace Engineering.

$\dagger$ Graduate Research Assistant, Department of Aerospace Engineering. Member AIAA. links of the system can be accomplished. Such additional flexibility in performing reorientation maneuvers should have great practical significance for completion of robotic tasks in space.

Another related application is the performance by astronauts of reorientation maneuvers in space. Although it is well known that astronauts in space can perform a variety of complicated reorientation maneuvers, without the use of thrusters, the theoretical basis for such maneuvers is incomplete. Again we note that an astronaut in space can be considered as a multibody system that satisfies all of the assumptions of this paper (except that motion is not restricted to be planar). Consequently, the theory in this paper is applicable in principle to the study of the maneuvering capability of astronauts in space. Previous research in this area ${ }^{9}$ has emphasized dynamics issues. Other closely related research has focused on describing the reorientation maneuvers of a falling cat. ${ }^{10}$

Finally, we mention another area of potential application of the results of this paper, namely, the development of deployment maneuvers for multibody antennas connected to a spacecraft. If deployment maneuvers for an antenna, or other deployable structures, are performed using only torque motors at the joints of the antenna segments, then the spacecraftantenna system is a multibody system that satisfies the assumptions of this paper. Consequently, our results can be used to develop efficient antenna deployment maneuvers. The importance of such deployment maneuvers is that they do not change the final orientation of the spacecraft or the total angular momentum of the spacecraft-antenna system, thereby reducing the requirements of the spacecraft momentum management system. To our knowledge, such control approaches to antenna deployment have not yet been exploited. It is expected that such an approach would have many advantages over the use of existing passive antenna deployment mechanisms. ${ }^{11}$

This paper is organized as follows. In Sec. II, a mathematical model for a planar multibody system in space is derived. We then formulate a control problem associated with planar multibody reorientation. In Sec. III, we first summarize several relevant theoretical results. We then introduce a control strategy to solve this reorientation problem. In Sec. IV, we apply the theoretical results to a three-link system. We present computer simulations illustrating the control strategy. Section $\mathrm{V}$ consists of a summary of the main results and concluding remarks about future research. Although a complete treatment of the topics in the paper requires use of differential geometric tools, our presentation avoids these tools and uses only elementary mathematical methods. However, references to relevant literature are provided throughout. 


\section{Problem Formulation}

We consider a system of $N$ planar rigid bodies interconnected by frictionless one-degree-of-freedom joints in the form of an open kinematic chain. The configuration space, for an observer at the center of mass of the system of rigid bodies, is $N$ dimensional. Since we assume an open kinematic chain, there are exactly $N-1$ joints. We consider controlling the rotational motion of the system using torques at the joints; each joint is assumed to be actuated so as to permit free adjustment of the joint angle. It is assumed that there are no external torques acting on the system. It is clear that the configuration of the $N$ bodies can be described by the absolute angle of any one of the bodies (say body 1 ) and $N-1$ joint angles. Denote by $\theta_{1}$ the absolute angle of body 1 and by the $(N-1)$ vector $\psi=\left(\psi_{1}, \ldots, \psi_{N-1}\right)$ the joint angle vector. Clearly, $\left(\theta_{1}, \psi\right)$ is a generalized coordinate vector for the rotational motion. It can be shown that the Lagrangian (which is equal to the rotational kinetic energy under the preceding assumptions), written in terms of these coordinates and their time derivatives, does not contain $\theta_{1}$ explicitly, i.e., $\theta_{1}$ is a cyclic or ignorable coordinate. Consequently, the generalized momentum associated with the cyclic coordinate $\theta_{1}$ is conserved. This conserved quantity is the first integral of the motion corresponding to conservation of angular momentum of the system. In this paper we assume zero initial angular momentum so that angular momentum remains zero throughout a maneuver.

It is clear that Lagrange's equations describe the motion on the joint angle space, and the evolution of $\theta_{1}$ can be obtained from the expression for conservation of angular momentum. Thus, the motion of a planar multibody system, under the preceding assumptions, can be described by the following reduced-order equations:

$$
\begin{gathered}
J_{s}(\psi) \ddot{\psi}+F_{s}(\psi, \dot{\psi})=\tau \\
\dot{\theta}_{1}+s^{\prime}(\psi) \dot{\psi}=0
\end{gathered}
$$

where $\tau=\left(\tau_{1}, \ldots, \tau_{N-1}\right)$ denotes the $(N-1)$ vector of joint torques, $J_{s}(\psi)$ is a symmetric positive definite $(N-1) \times(N-1)$ matrix function, and $s(\psi)$ and $F_{s}(\psi, \dot{\psi})$ are $(N-1)$ vector functions. Note that in this paper a prime denotes transpose. The explicit specifications of these functions can be found in the literature. ${ }^{1,2,12}$

State-space equations for Eqs. (1) and (2) are

$$
\begin{gathered}
\dot{\theta}_{1}=-s(\psi)^{\prime} \omega \\
\dot{\psi}=\omega \\
\dot{\omega}=-J_{s}^{-1}(\psi) F_{s}(\psi, \omega)+J_{s}^{-1}(\psi) \tau
\end{gathered}
$$

Note that Eqs. (4) and (5) are expressed in terms of the joint phase variables $(\psi, \dot{\psi})$ only. Hence the joint angle space constitutes a reduced configuration space for the system. This reduced configuration space is also referred to as the "shape space" of the system. ${ }^{12-16}$ It is possible to consider control problems expressed solely in terms of the shape space; such problems can be solved using classical methods. However, in our work we are interested in the more general control problems associated with the complete dynamics of the multibody system defined by Eqs. (1) and (2) [or Eqs. (3-5)] .

Note that Eqs. (4) and (5) only, which represent the projection of the motion onto the shape phase space, are feedback linearizable using the feedback transformation

$$
u=-J_{s}^{-1}(\psi) F_{s}(\psi, \omega)+J_{s}^{-1}(\psi) \tau
$$

where $u \in R^{N-1}$. The previous feedback transformation yields the following normal form equations:

$$
\dot{\theta}_{1}=-s(\psi)^{\prime} \omega
$$

$$
\begin{aligned}
& \dot{\psi}=\omega \\
& \dot{\omega}=u
\end{aligned}
$$

We remark here that it is impossible to completely linearize the system defined by Eqs. (3-5) using static or dynamic feedback combined with any coordinate transformation.

Note that an equilibrium solution of Eqs. (3-5) corresponding to $\tau=0$ [or equivalently an equilibrium solution of Eqs. (7-9) for $u=0$ ] is given by $\left(\theta_{1}^{e}, \psi^{e}, 0\right)$, where $\left(\theta_{1}^{e}, \psi^{e}\right)$ is referred to as an equilibrium configuration. Hence, an equilibrium solution corresponds to a trivial motion of the system for which all of the configuration space variables remain constant.

Note also that Eq. (3) represents conservation of angular momentum. This equation is nonholonomic for $N \geq 3$ (i.e., if the multibody system consists of three or more links), since the differential expression (3) is not integrable for $N \geq 3$. This fact has important implications in terms of controllability properties of the system as will be shown in the subsequent development. As a consequence of the symmetry possessed by the system, $\theta_{1}$ does not apear explicitly in Eq. (3). Mechanical systems with such symmetry properties are referred to as nonholonomic Caplygin systems. ${ }^{17-21}$ As a consequence of the nonintegrability for $N \geq 3$, the scalar analytic functions

$$
H_{i j}(\psi)=\frac{\partial s_{i}(\psi)}{\partial \psi_{j}}-\frac{\partial s_{j}(\psi)}{\partial \psi_{i}}, \quad(i, j) \in I^{2}
$$

where $I=\{1, \ldots, N-1\}$, do not all vanish, except possibly on a set that has measured zero with respect to the shape space.

\section{Reorientation Maneuvering Problem}

In this section, we address the following control problem associated with planar multibody systems described by Eqs. (1) and (2):

Problem: Given an initial state $\left(\theta_{1}^{0}, \psi^{0}, \omega^{0}\right)$ and a desired equilibrium solution $\left(\theta_{1}^{e}, \psi^{e}, 0\right)$, determine a motion

$$
\left[\theta_{1}(t), \psi(t), \omega(t)\right], \quad 0 \leq t \leq t_{f}
$$

such that

$$
\begin{gathered}
\left(\theta_{1}(0), \psi(0), \omega(0)\right)=\left(\theta_{1}^{0}, \psi^{0}, \omega^{0}\right), \quad\left(\theta_{1}\left(t_{f}\right)\right. \\
\left.\psi\left(t_{f}\right), \quad \omega\left(t_{f}\right)\right)=\left(\theta_{1}^{e}, \psi^{e}, 0\right)
\end{gathered}
$$

and

$$
\left(\theta_{1}(t), \psi(t), \omega(t)\right)
$$

satisfies Eqs. (1) and (2) for some control function $t \rightarrow \tau(t)$.

Note that, in particular, if $\omega^{0}=0$, then this problem corresponds to a rest-to-rest maneuver.

The existence of solutions to this control problem was demonstrated in our earlier work. ${ }^{1,2}$ In particular, we studied the nonlinear control system described by Eqs. (7-9) and employed certain results from nonlinear control theory to characterize controllability properties of planar multibody systems described by Eqs. (1) and (2). These results not only prove the existence of solutions of the preceding problem but also provide a theoretical basis for construction of nonlinear control strategies required to achieve the desired maneuver. We next summarize those results..$^{1,2}$

Under the stated assumptions, a planar multibody system has the following properties if $N \geq 3$, i.e., if it consists of three or more links:

1) The system is strongly accessible.

2) The system is small time locally controllable from any equilibrium.

3) The system can be transferred from any initial condition to any desired equilibrium in arbitrarily small time. 
If $N=1$ or 2 , then the system is not even accessible and is not small time locally controllable, and there exist initial conditions that cannot be transferred to a desired equilibrium.

The proofs ${ }^{1,19}$ of the first two results depend on showing that certain Lie algebraic conditions are satisfied if $N \geq 3$. The third result is proved ${ }^{1,19}$ constructively.

It should be emphasized that the subsequent development is assumed to be carried out for multibody systems consisting of three or more links $(N \geq 3)$; this should be understood even if it is not always explicitly stated. Note that the reorientation problem generally has many solutions. In this paper, we describe one solution approach, outline the theory behind it, and present some data from simulations. The key observation is the following.

Consider Eq. (3). Assume that joint angles are controlled in such a way that $\psi(t), 0 \leq t_{1} \leq t \leq t_{2}$, describes a closed path $\gamma$ in the shape space. Integrating both sides of Eq. (3) from $t=t_{1}$ to $t=t_{2}$ and using the fact that $\mathrm{d} \psi=\dot{\psi} \mathrm{d} t$, we obtain

$$
\theta_{1}\left(t_{2}\right)-\theta_{1}\left(t_{1}\right)=\oint_{\gamma} s^{\prime}(\psi) \mathrm{d} \psi
$$

Thus, by proper selection of a path $\gamma$ in shape space, any desired geometric phase (which is a rotation of link 1) can be obtained. By the nonintegrability property just mentioned, the preceding integral is in fact path dependent, thereby guaranteeing the existence of (many) such paths.

Note that in differential geometry the quantity

$$
\alpha(\gamma)=\oint_{\gamma} s^{\prime}(\psi) \mathrm{d} \psi
$$

is referred to as the geometric phase (or holonomy) of the closed path $\gamma$. This quantity depends only on the geometry of the closed path and is independent of the speed at which the path is traversed.

Note that Stokes' formula can be applied to obtain an equivalent formula for $\alpha(\gamma)$ as a surface integral. For simplicity, assume that $N=3$, i.e., the shape space is the $\left(\psi_{1}, \psi_{2}\right)$ plane. Also, let $\gamma$ be traversed counterclockwise. Then by Stokes' theorem the preceding formula can be written as

$$
\alpha(\gamma)=\int_{S}\left(\frac{\partial s_{2}}{\partial \psi_{1}}-\frac{\partial s_{1}}{\partial \psi_{2}}\right) \mathrm{d} \psi_{1} \mathrm{~d} \psi_{2}
$$

where $S$ is the surface within the boundary $\gamma$. In the case that the path is traversed clockwise, the surface integral is equal to $-\alpha(\gamma)$.

More information concerning geometric phases can be found in the literature. ${ }^{15}$ Geometric phase ideas have proved useful in a variety of inherently nonlinear control problems. ${ }^{19-21}$ These ideas have also been used for a class of path planning problems based solely on kinematic relations. ${ }^{13,14,16}$

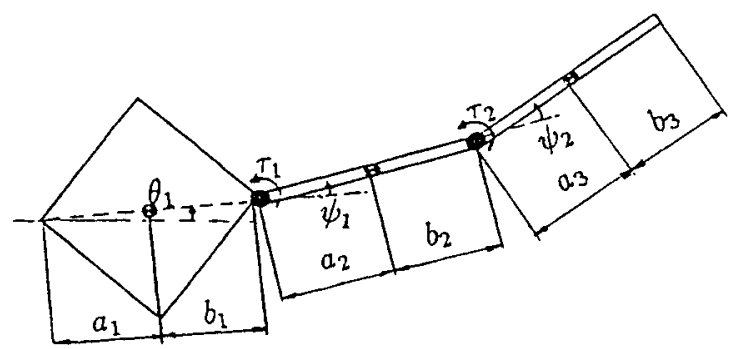

Fig. 1 Three-link example.

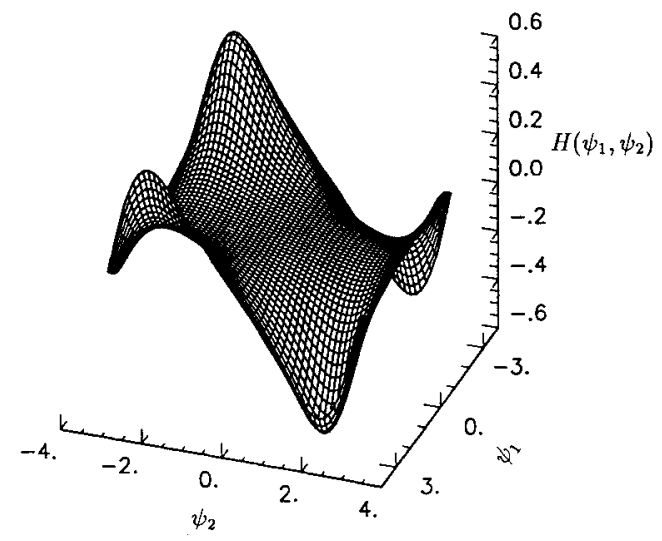

Fig. 2 Function $\boldsymbol{H}\left(\psi_{1}, \psi_{2}\right)$.

We now describe a control strategy, using the preceding geometric phase relation (11), which solves the reorientation problem.

Let $\left(\theta_{1}^{e}, \psi^{e}, 0\right)$ denote the desired equilibrium solution. We refer to $\left(\theta_{1}^{e}, \psi^{e}\right)$ and $\psi^{e}$ as the desired equilibrium configuration and the desired equilibrium shape, respectively. We describe four steps involved in construction of an open-loop control function $u_{\left[0, t_{f}\right)}=\left(u_{1}, \ldots, u_{N-1}\right)^{\prime}$ that transfers any initial state $\left(\theta_{1}^{0}, \psi^{0}, \omega^{0}\right)$ to $\left(\theta_{1}^{e}, \psi^{e}, 0\right)$ in time exactly $t_{f}$, where $t_{f}>0$ is arbitrary.

Let $0<t_{1}<t_{2}<t_{3}<t_{f}$ denote an arbitrary partition of the time interval $\left[0, t_{f}\right)$.

Step 1: Transfer the system to the desired equilibrium shape, i.e., find a control that transfers the initial state $\left(\theta_{1}^{0}, \psi^{0}, \omega^{0}\right)$ to $\left(\theta_{1}^{1}, \psi^{e}, 0\right)$ at time $t_{1}$, for some $\theta_{1}^{1}$.

Since the dynamics on the shape phase space are so simple, namely, decoupled double integrators, step 1 has many solutions that are easily obtained using classical methods. One such control function is

$$
u_{\left[0, t_{1}\right)}= \begin{cases}-\frac{\pi \omega^{0}}{t_{1}} \cos \left(\frac{\pi t}{t_{1}}\right) & t \in\left[0,0.5 t_{1}\right) \\ \frac{8 \pi\left[\psi^{e}-\psi^{0}-\omega^{0} t_{1}\left(0.5-\pi^{-1}\right)\right]}{t_{1}^{2}} \sin \left[\frac{2 \pi\left(2 t-t_{1}\right)}{t_{1}}\right] & t \in\left[0.5 t_{1}, t_{1}\right)\end{cases}
$$

Next, we select a closed path $\gamma$ (or a series of closed paths, see remark 1 following) in the shape space that achieves the desired geometric phase. There are many ways to accomplish such a construction; in our work we have found it convenient to use only two joint motions, keeping the other joints locked, and to use a square path in the restricted two-dimensional shape space. It is convenient to select the center of the square path in a region of the shape space that corresponds to a "large" geometric phase change (see remark 2 following).

To make the earlier ideas more concrete, we present a specific construction. Let $(i, j) \in I^{2}, i \neq j$, denote a pair of joints. Assume that for $t \geq t_{1}$ only this pair of joints are actuated while all of the other joints are kept fixed. This is equivalent to locking all of the joints except the ones labeled $i$ and $j$ and treating the $N$ bodies as three interconnected bodies, for $t \geq t_{1}$. In this case the desired geometric phase formula can be written as

$$
\theta_{1}\left(t_{f}\right)-\theta_{1}^{1}= \pm \alpha(\gamma)
$$

where $+(-)$ corresponds to counterclockwise (clockwise) traversal of the closed path $\gamma$. Since we desire to make $\theta_{1}\left(t_{f}\right)=\theta_{1}^{e}$, the closed path $\gamma$ should be selected to satisfy 


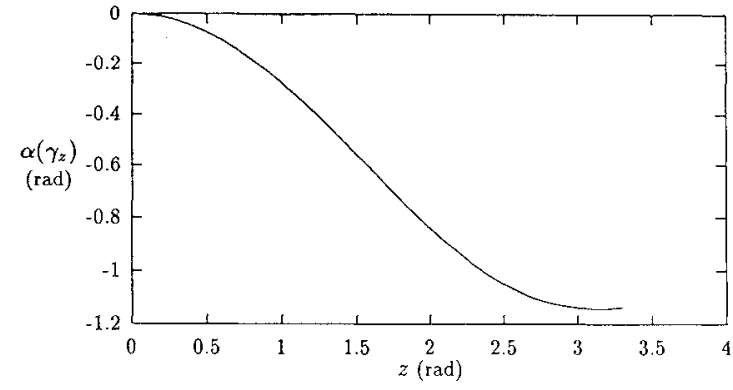

Fig. 3 Geometric phase curve.

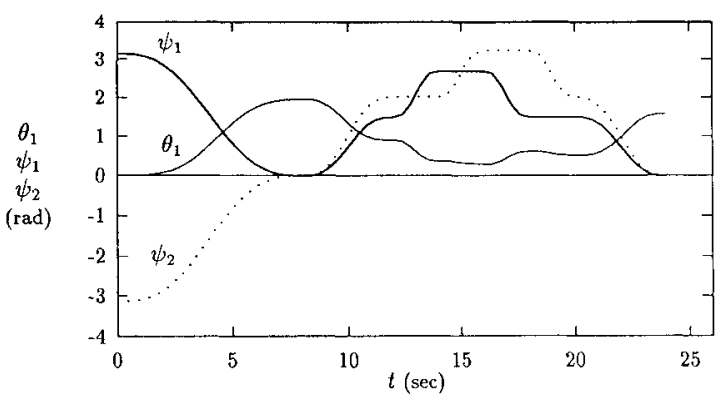

Fig. 4 Time responses for $\theta_{1}, \psi_{1}$, and $\psi_{2}$.

The path $\gamma$ lies in the two-dimensional $\left(\psi_{i}, \psi_{j}\right)$ plane, so that

$$
\alpha(\gamma)=\oint_{\gamma} \tilde{s}_{i}\left(\psi_{i}, \psi_{j}\right) \mathrm{d} \psi_{i}+\tilde{s}_{j}\left(\psi_{i}, \psi_{j}\right) \mathrm{d} \psi_{j}
$$

where the scalar functions $\tilde{s}_{i}\left(\psi_{i}, \psi_{j}\right)$ and $\tilde{s}_{j}\left(\psi_{i}, \psi_{j}\right)$ are obtained by evaluating $s_{i}(\psi)$ and $s_{j}(\psi)$ at $\psi_{k}=\psi_{k}^{e}, \forall k \in I$ where $k \neq i, j$.

As mentioned earlier, we choose $\gamma$ to be a square path in the $\left(\psi_{i}, \psi_{j}\right)$ plane that is centered at the shape defined by $\psi^{*}$ and that has side of length $z^{*}$, where $z^{*}$ satisfies

$$
\pm \alpha\left(\gamma_{z^{*}}\right)+\theta_{1}^{1}-\theta_{1}^{e}=0
$$

Here $\gamma_{z}$ indicates the dependence of the square path on the size parameter $z$. In most cases, this equation is easily solved using standard numerical procedures.

Thus the four corner points of this square path are defined by shape vectors

$$
\begin{aligned}
& p_{1}^{*}=\psi^{*}-0.5 z^{*}\left(e_{i}+e_{j}\right) \\
& p_{2}^{*}=\psi^{*}+0.5 z^{*}\left(e_{i}-e_{j}\right) \\
& p_{3}^{*}=\psi^{*}+0.5 z^{*}\left(e_{i}+e_{j}\right) \\
& p_{4}^{*}=\psi^{*}-0.5 z^{*}\left(e_{i}-e_{j}\right)
\end{aligned}
$$

where $e_{i}$ and $e_{j}$ are the $i$ th and $j$ th standard basis vectors in $R^{N-1}$. Thus the specific square path selected depends on the $N-1$ vector $\psi^{*}$ that is the center of the square and the size of the square $z^{*}$.

Remark 1: Note that here, for notational simplicity in presenting the main idea, we assume that the desired geometric phase can be obtained by a single closed path. In general, more than one closed path may be required to produce the desired geometric phase; for such cases $\gamma$ can be viewed as a concatenation of a series of closed paths.

Remark 2: Selection of the center point $\psi^{*}$ of the path is rather arbitrary, e.g., one selection is $\psi^{*}=\psi^{e}$. However, other choices may provide a greater change in the geometric phase for a given size path. In this regard, the use of Stokes' theo- rem, as indicated previously, suggests that $\psi^{*}$ should be chosen where

$$
\left|\frac{\partial s_{j}(\psi)}{\partial \psi_{i}}-\frac{\partial s_{i}(\psi)}{\partial \psi_{j}}\right|
$$

is a maximum.

We now describe the remaining three steps as follows.

Step 2: Transfer the system from state $\left(\theta_{1}^{1}, \psi^{e}, 0\right)$ to a state corresponding to the corner of $\gamma$ closest to $\psi^{e}$, along an arbitrary path in the shape space, in $t_{2}-t_{1}$ units of time.

As an example, if $p_{1}^{*}$ is the corner of $\gamma$ closest to $\psi^{e}$, we propose the following control function for step 2:

$$
u_{\left[t_{1}, t_{2}\right)}=\frac{2 \pi\left(p_{1}^{*}-\psi^{e}\right)}{\left(t_{2}-t_{1}\right)^{2}} \sin \left[\frac{2 \pi\left(t-t_{1}\right)}{\left(t_{2}-t_{1}\right)}\right]
$$

Step 3: Traverse the selected square path (counterclockwise or clockwise, depending on the sign of the desired geometric phase value), in $t_{3}-t_{2}$ units of time; the resulting change in the angle $\theta_{1}$ is necessarily $\theta_{1}^{e}-\theta_{1}^{1}$.

Without loss of generality, we assume that the desired geometric phase value is obtained by counterclockwise traversal of the closed path starting and ending at $p_{1}^{*}$. Then, the following control functions guarantee traversal of the closed path, thereby accomplishing step 3:

$$
\begin{aligned}
u_{\left[t_{2}, t_{2}+h\right)} & =\frac{2 \pi\left(p_{2}^{*}-p_{1}^{*}\right)}{h^{2}} \sin \left[\frac{2 \pi\left(t-t_{2}\right)}{h}\right] \\
u_{\left[t_{2}+h, t_{2}+2 h\right)} & =\frac{2 \pi\left(p_{3}^{*}-p_{2}^{*}\right)}{h^{2}} \sin \left[\frac{2 \pi\left(t-t_{2}-h\right)}{h}\right] \\
u_{\left[t_{2}+2 h, t_{2}+3 h\right)} & =\frac{2 \pi\left(p_{4}^{*}-p_{3}^{*}\right)}{h^{2}} \sin \left[\frac{2 \pi\left(t-t_{2}-2 h\right)}{h}\right] \\
u_{\left[t_{2}+3 h, t_{3}\right)} & =\frac{2 \pi\left(p_{1}^{*}-p_{4}^{*}\right)}{h^{2}} \sin \left[\frac{2 \pi\left(t-t_{2}-3 h\right)}{h}\right]
\end{aligned}
$$

where $h=\left(t_{3}-t_{2}\right) / 4$.

Step 4: Transfer the system back to the desired equilibrium shape $\psi^{e}$ following the path used in step 2 , in $t_{f}-t_{3}$ units of time, thereby guaranteeing that the desired final state $\left(\theta_{1}^{e}, \psi^{e}, 0\right)$ is reached at time $t_{f}$.

The following control function

$$
u_{\left[t_{3}, t_{f}\right)}=\frac{2 \pi\left(\psi^{e}-p_{1}^{*}\right)}{\left(t_{f}-t_{3}\right)^{2}} \sin \left[\frac{2 \pi\left(t-t_{3}\right)}{\left(t_{f}-t_{3}\right)}\right]
$$

accomplishes step 4 .

The corresponding control torque $\tau$ can be computed using Eq. (6). It is clear that the constructed control torque transfers the initial condition of the system (1) and (2) to the desired equilibrium configuration at time $t_{f}$. It is important to emphasize that the preceding construction is based on a priori selection of a square as the closed path in the shape space. Selection of square paths simplifies computation of the controls; how-

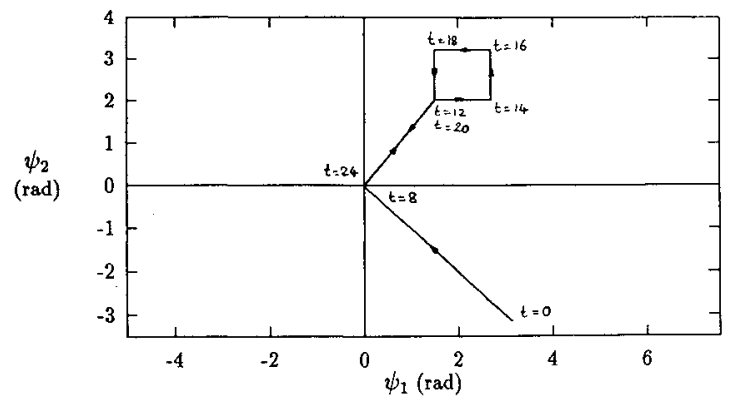

Fig. 5 Motion in shape space. 
Table 1 System characteristics

\begin{tabular}{ccccc}
\hline Body $i$ & $a, \mathrm{~m}$ & $b, \mathrm{~m}$ & $m, \mathrm{~kg}$ & $I, \mathrm{~kg}-\mathrm{m}^{2}$ \\
\hline 1 & 0.5 & 0.5 & 120 & 10 \\
2 & 0.5 & 0.5 & 12 & 1 \\
3 & 0.5 & 0.5 & 12 & 1 \\
\hline \hline
\end{tabular}

ever, other path selections could be made. There are infinitely many choices for control functions that accomplish the preceding four steps, and the total time required is arbitrary.

\section{Example of Maneuvering a Three-Body System}

In this section, the theory developed in Sec. III is used to illustrate a specific maneuver for interconnected multibodies using only torque inputs at the joint connections. As discussed, general planar maneuvers cannot be achieved using two or fewer interconnected links. An interconnection of three links provides complete maneuvering capability; consequently, that is the case considered here. Maneuvers of an interconnection of more than three links can always be reduced to a sequence of submaneuvers, each submaneuver involving the motion of only three links.

For illustration purposes we consider a planar three-link system modeled as in Fig. 1. The first link represents a spacecraft, whereas the other two links represent antenna segments; the reorientation maneuver that is studied represents a deployment of the antenna that is to be accomplished while achieving a specified orientation of the spacecraft. The system characteristics are given in Table 1.

Using the notation already introduced with $N=3$, the following are the reduced-order equations of motion

$$
\begin{gathered}
\dot{\theta}_{1}=s_{1}\left(\psi_{1}, \psi_{2}\right) \omega_{1}+s_{2}\left(\psi_{1}, \psi_{2}\right) \omega_{2} \\
\dot{\psi}_{1}=\omega_{1} \\
\dot{\psi}_{2}=\omega_{2} \\
\dot{\omega}_{1}=u_{1} \\
\dot{\omega}_{2}=u_{2}
\end{gathered}
$$

The functions $s_{1}\left(\psi_{1}, \psi_{2}\right)$ and $s_{2}\left(\psi_{1}, \psi_{2}\right)$, determined from the angular momentum expression, are given as

$$
s_{i}(\psi)=-N_{i}(\psi) / D(\psi), \quad i=1,2
$$

where

$$
\begin{aligned}
& N_{1}(\psi)=17.5+7.5 \cos \psi_{1}+10.5 \cos \psi_{2}+2.5 \cos \left(\psi_{1}+\psi_{2}\right) \\
& N_{2}(\psi)=3.75+5.25 \cos \psi_{2}+2.5 \cos \left(\psi_{1}+\psi_{2}\right) \\
& D(\psi)=32.5+15 \cos \psi_{1}+10.5 \cos \psi_{2}+5 \cos \left(\psi_{1}+\psi_{2}\right)
\end{aligned}
$$

and the transformed input $u$ is related to the control torque $\tau$ by

$$
u=-J_{s}^{-1}(\psi) F_{s}(\psi, \omega)+J_{s}^{-1}(\psi) \tau
$$

where $J_{s}(\psi)$ is a $2 \times 2$ matrix with entries

$$
\begin{aligned}
& J_{s_{11}}(\psi)=17.5+10.5 \cos \psi_{2}-N_{1}^{2}(\psi) / D(\psi) \\
& J_{s_{12}}(\psi)=3.75+5.25 \cos \psi_{2}-N_{2}^{2}(\psi) / D(\psi) \\
& J_{s_{21}}(\psi)=3.75+5.25 \cos \psi_{2}-N_{2}^{2}(\psi) / D(\psi) \\
& J_{s_{22}}(\psi)=3.75-N_{2}^{2}(\psi) / D(\psi)
\end{aligned}
$$

and the vector function $F_{s}(\psi, \omega)$ can be expressed as

$$
F_{s}(\psi, \omega)=\frac{\mathrm{d} J_{s}(\psi)}{\mathrm{d} t} \omega-\frac{1}{2} \frac{\partial}{\partial \psi}\left[\omega^{\prime} J_{s}(\psi) \omega\right]
$$

where $\psi=\left(\psi_{1}, \psi_{2}\right)$ and $\omega=\left(\omega_{1}, \omega_{2}\right)$. We first compute the function

$$
H(\psi)=\frac{\partial s_{2}(\psi)}{\partial \psi_{1}}-\frac{\partial s_{1}(\psi)}{\partial \psi_{2}}
$$

on $[-\pi, \pi] \times[-\pi, \pi]$. This function is shown graphically in Fig. 2. The joint angles $\left(\psi_{1}, \psi_{2}\right)$, where $H$ takes the largest absolute value, are approximately

$$
[(2 \pi / 3,5 \pi / 6),(-5 \pi / 6,-2 \pi / 3)]
$$

Consequently, geometric phases for the square paths centered at $\psi^{*}=(2 \pi / 3,5 \pi / 6)$ are computed numerically. Figure 3 shows the geometric phase as a function of the size of the square path.

We present a representative rest-to-rest maneuver that deploys the antenna segments from a folded configuration to a deployed configuration while achieving a desired orientation of the spacecraft link. The maneuver is defined by an initial rest configuration $(0, \pi,-\pi)$ and a final rest configuration

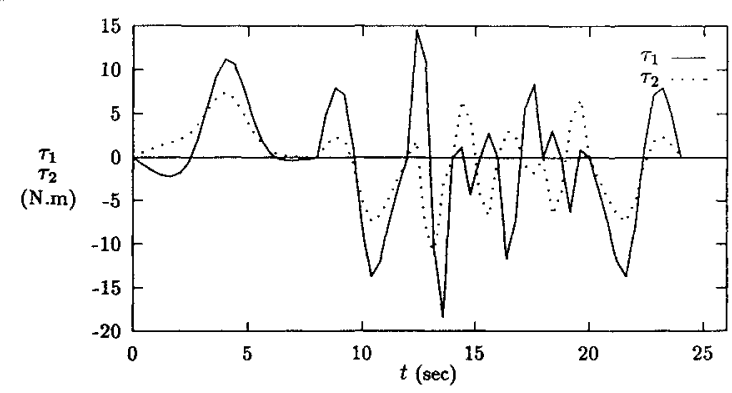

Fig. 6 Control torques $\tau_{1}$ and $\tau_{2}$.

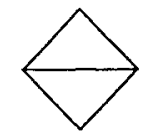

Initial Configuration

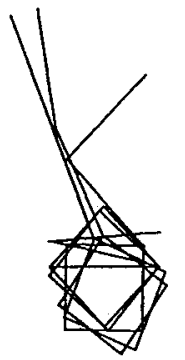

Step 1

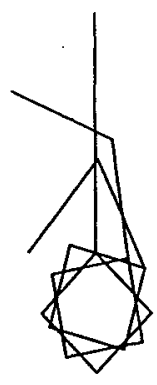

Step 4

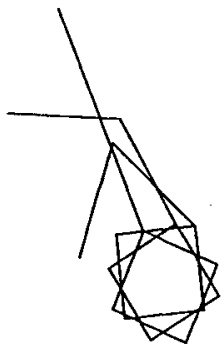

Step 2

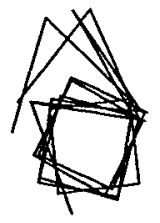

Step 3
Fig. 7 Configuration of links. 
$(0.5 \pi, 0,0)$. The specific control functions indicated previously were used in the simulation; the times for each of the indicated steps are $t_{1}=8, t_{2}=12, t_{3}=20$, and $t_{f}=24$. In this particular case, the required geometric phase change $\theta_{1}^{e}-\theta_{1}^{1}$ was computed to be $0.39 \mathrm{rad}$, which defined the square path used in the simulation.

The time responses for $\theta_{1}, \psi_{1}$, and $\psi_{2}$ are shown in Fig. 4. Figure 5 illustrates the motion in the shape space. The control torques $\tau_{1}$ and $\tau_{2}$ are shown in Fig. 6. In Fig. 7 the maneuver is demonstrated by showing the configuration of the links for a sequence of uniformly spaced time instants.

\section{Conclusions}

In this paper we have developed a reorientation maneuvering strategy for planar rigid bodies interconnected by ideal pin joints in the form of an open kinematic chain. The maneuver strategy uses the nonintegrability of the expression for angular momentum conservation. We have demonstrated that large angle maneuvers can be designed to achieve an arbitrary reorientation of the multibody system with respect to an inertial frame; the maneuvers are performed using internal controls, e.g., servo torque motors located at the joints of the body segments. The theoretical background for carrying out the required maneuvers has been briefly summarized. The results have been applied to a specific space maneuver of a three-body interconnection. We mention two nontrivial extensions of the approach in this paper that are currently being developed. The first extension is to nonplanar reorientation maneuvers of multibody systems; in this case the dynamics issues are much more complicated, but in principle the approach is viable. ${ }^{22}$ Another extension is the development of feedback implementations of the controls presented in this paper; some results have been obtained ${ }^{19}$ using a (necessarily) discontinuous feedback strategy. These important extensions generally require the use of differential geometric methods for a complete treatment. One motivation of the present paper has been to present the key ideas, in the case of planar reorientation maneuvers, using only elementary methods of analysis.

\section{Acknowledgments}

The authors acknowledge financial support for their work from NSF under Grant DMS-9002136 and from NASA under Grant NAG-1-1419.

\section{References}

${ }^{1}$ Reyhanoglu, M., and McClamroch, N. H., "Controllability and Stabilizability of Planar Multibody Systems with Angular Momentum Preserving Control Torques," Proceedings of the American Control Conference (Boston, MA), Inst. of Electrical and Electronics Engineers, Piscataway, NJ, 1991, pp. 1102-1107.

${ }^{2}$ Reyhanoglu, M., and McClamroch, N. H., "Reorientation of Space Multibody Systems Maintaining Zero Angular," Proceedings of the AIAA Guidance, Navigation, and Control Conference (New Orleans, LA), AIAA, Washington, DC, 1991, pp. 1330-1340.

${ }^{3}$ Spofford, J. R., and Akin, D. L., "Redundancy Control of a Free-Flying Telerobot," Journal of Guidance, Control, and Dynamics, Vol. 13, No. 3, 1990, pp. 515-523.

${ }^{4}$ Longman, R. W., Lindberg, R. E., and Zedd, M. F., "Satellite Mounted Robot Manipulators-New Kinematics and Reaction Mo- mentum Compensation," International Journal of Robotics Research, Vol. 6, 1987, pp. 87-103.

${ }^{5}$ Papadopoulos, E., and Dubowsky, S., "On the Nature of Control Algorithms for Space Manipulators," Proceedings of the Conference on Robotics and Automation (Cincinnati, $\mathrm{OH}$ ), Inst. of Electrical and Electronics Engineers, Piscataway, NJ, 1990, pp. 1102-1108.

${ }^{6}$ Yoshida, K., and Umetani, Y., "Control of Space Free-Flying Robot," Proceedings of the IEEE Conference on Decision and Control (Honolulu, HI), Inst. of Electrical and Electronics Engineers, Piscataway, NJ, 1990, pp. 97-102.

${ }^{7}$ Luo, Z. H., and Sakawa, Y., "Control of Space Manipulator for Capturing a Tumbling Object,"' Proceedings of the IEEE Conference on Decision and Control (Honolulu, HI), Inst. of Electrical and Electronics Engineers, Piscataway, NJ, 1990, pp. 103-107.

${ }^{8}$ Fujii, H., Murayama, T., Nakajima, K., and Anazawa, S., "Capture Control for Manipulator Arm of Free-Flying Space Robot," Proceedings of the AIAA Guidance, Navigation, and Control Conference (Portland, OR), AIAA, Washington, DC, 1990.

${ }^{9}$ Kane, T. R., and Scher, M. P., "Human Self-Rotation By Means of Limb Movements," Journal of Biomechanics, Vol. 3, 1970, pp. 39 49.

${ }^{10}$ Kane, T. R., and Scher, M. P., "A Dynamical Explanation of the Falling Cat Phenomenon," International Journal of Solids and Structures, Vol. 5, 1969, pp. 663-670.

${ }^{11}$ Misawa, M., Yasaka, T., and Miyake, S., "Analytical and Experimental Investigations for Satellite Antenna Deployment Mechanisms," Journal of Spacecraft and Rockets, Vol. 26, No. 3, 1989, pp. 181-187.

${ }^{12}$ Sreenath, N., "Nonlinear Control of Multibody Systems in Shape Space," Proceedings of the Conference on Robotics and Automation (Cincinnati, $\mathrm{OH}$ ), Inst. of Electrical and Electronics Engineers, Piscataway, NJ, 1990, pp. 1776-1781.

${ }^{13} \mathrm{Li}, \mathrm{Z}$., and Canny, J., "Motion of Two Rigid Bodies with Rolling Constraint," IEEE Transactions on Robotics and Automatic, Vol. 6, No. 1,1990 , pp. 62-71.

${ }^{14} \mathrm{Li}, \mathrm{Z}$, and Montgomery, R., "Dynamics and Optimal Control of a Legged Robot in Flight Phase," Proceedings of the Conference on Robotics and Automation (Cincinnati, $\mathrm{OH}$ ), Inst. of Electrical and Electronics Engineers, Piscataway, NJ, 1990, pp. 1816-1821.

${ }^{15}$ Marsden, J., Montgomery, R., and Ratiu, T., "Reduction, Symmetry and Phases in Mechanics," Memoirs of the American Mathematical Society, American Mathematical Society, Providence, RI, 1990.

${ }^{16}$ Krishnaprasad, P. S., "Geometric Phases and Optimal Reconfiguration for Multibody Systems,"' Proceedings of the American Control Conference (San Diego, CA), 1990, pp. 2440-2444.

${ }^{17}$ Arnold, V. I., Dynamical Systems III, Vol. 3, Springer-Verlag, New York, 1988

${ }^{18}$ Neimark, J. I., and Fufaev, F. A., Dynamics of Nonholonomic Systems, Vol. 33, Translations of Mathematical Monographs, American Mathematical Society, Providence, RI, 1972.

${ }^{19}$ Bloch, A. M., Reyhanoglu, M., and McClamroch, N. H., "Control and Stabilization of Nonholonomic Dynamic Systems," IEEE Transactions on Automatic Control (to be published).

${ }^{20}$ Bloch, A. M., Reyhanoglu, M., and McClamroch, N. H., "Control and Stabilization of Nonholonomic Caplygin Dynamic Systems,", Proceedings of the IEEE Conference on Decision and Control (Brighton, England, UK), Inst. of Electrical and Electronics Engineers, Piscataway, NJ, 1991, pp. 1127-1132.

${ }^{21}$ Reyhanoglu, M., McClamroch, N. H., and Bloch, A. M., "Motion Planning for Nonholonomic Dynamic Systems," Recent Advances in Nonholonomic Motion Planning, Kluwer Publishers (to be published).

${ }^{22}$ Walsh, G. C., and Sastry, S., "On Reorienting Linked Rigid Bodies Using Internal Motions," Proceedings of the IEEE Conference on Decision and Control (Brighton, England, UK), Inst. of Electrical and Electronics Engineers, Piscataway, NJ, 1991, pp. 1190-1195. 